\title{
Massive Cryptosporidium infections and chronic diarrhea in HIV-negative patients
}

\author{
Łukasz Pielok $^{1}$ (1) Szymon Nowak ${ }^{1} \cdot$ Matylda Kłudkowska ${ }^{1,2} \cdot$ Krystyna Frąckowiak $^{2} \cdot$ Łukasz Kuszel $^{3} \cdot$ Paweł Zmora $^{4}$. \\ Jerzy Stefaniak ${ }^{1}$
}

Received: 11 September 2018 / Accepted: 25 March 2019/Published online: 12 April 2019

(C) The Author(s) 2019

\begin{abstract}
Protozoa of the genus Cryptosporidium are common parasites of domestic and wild animals - mammals, birds, reptiles, and fishes. The invasive forms are thick-walled oocysts, which can be present in water supplies, on fruits, vegetables, or in the soil contaminated with feces. In this work, we describe three cases of middle-aged persons with massive Cryptosporidium hominis infection and chronic diarrhea with no immunological abnormalities and no history of previous travels to tropical countries. The lesions discovered during colonoscopy within the large intestine-cryptitis and the histopathological changes were related to massive cryptosporidiosis. All these statements indicate necessity of parasitological stool examination in cases with chronic diarrhea in which no etiological agents are detected, but not only in HIV positive individuals. Parasite's eradication leads to symptom disappearance as well as improvement of histopathological mucosa alterations.
\end{abstract}

Keywords Cryptosporidium spp. $\cdot$ Cryptosporidiosis $\cdot$ Diarrhea $\cdot$ Chronic diarrhea $\cdot$ Cryptitis $\cdot$ Nitazoxanide $\cdot$ Immunocompetent

\section{Introduction}

Protozoa of the genus Cryptosporidium are common parasites of domestic and wild animals - mammals, birds, reptiles, and fishes (Zehedi et al. 2015; Osman et al. 2017; Yu et al. 2018). The invasive forms for all hosts are thick-walled oocysts, strongly resistant for chlorine disinfection, which can be present in water supplies, on fruits, vegetables, or in the soil contaminated with feces (Toledo et al. 2017; Squire and Ryan 2017; Domenech et al. 2018). The most important way of transmission of these parasites is the fecal-oral route, by

Section Editor: Panagiotis Karanis

Łukasz Pielok

1.augustyn@wp.pl

1 Department and Clinic of Tropical and Parasitic Diseases, Poznań University of Medical Sciences, Przybyszewskiego Street, 60-355 Poznań, Poland

2 Central Laboratory of Microbiology, H. Święcicki University Hospital, Poznań, Poland

3 Department of Medical Genetics, Poznań University of Medical Sciences, Poznań, Poland

4 Institute of Bioorganic Chemistry Polish Academy of Sciences, Poznań, Poland swallowing oocysts with contaminated food or nontreated water or during recreational water events (lakes, rivers, and swimming pools) (Hall et al. 2017; Fill et al. 2017; Hlavsa et al. 2017). The presence of Cryptosporidium spp. and Giardia intestinalis in waste water is a main concern because water reuse for irrigation can jeopardize human health (Domenech et al. 2018). The direct way of transmission from person to person of these apicomplexan parasites is also possible, that is why human, especially asymptomatic carrier, becomes a possible source of infection for his surroundings, creating a serious epidemiological threat.

Cryptosporidium trophozoites exist in the enterocytes and are responsible for their damage causing gastrointestinal symptoms such as watery diarrhea and abdominal cramps (Bouzid et al. 2013). The pathologic process in an immunocompetent host is usually self-limiting, but sometimes, protracted infections can be present. In such cases, chronic diarrhea lasts longer than 4-6 weeks (Sandhu and Surawicz 2012). Persistent intestinal disorders are present mainly in persons suffering from different types of immunodeficiency. Cryptosporidiosis is classically reported in patients with acquired immunodeficiency syndrome and emerged as a cause of persistent diarrhea in solid transplant patients (Kaniyarakkal et al. 2016; DuPont 2016). Cryptosporidiosis is also a late post-transplant infection that can disseminate to biliary ducts or lungs (Lantermier et al. 2017). In HIV-infected 
patients, it is an opportunistic infection - an indicator of full symptomatic AIDS (Shrivastava et al. 2017a, 2017b).

In this work we describe three cases of middle-aged persons with massive Cryptosporidium spp. infection and chronic diarrhea with no immunological abnormalities.

\section{Case 1}

Forty-six-year-old male admitted to the Clinic of Tropical and Parasitic Diseases, Poznan, Poland, because of persistent lowgrade fever, lymphadenopathy, joint pains, and watery diarrhea lasting for 5 weeks. Prior to the admission, he was hospitalized in the Internal Ward, but no tentative diagnosis was established, except chronic tonsillitis (he was classified among surgery treatment). Previously, he was also suffered from skin Streptococcus pyogenes and Staphylococcus aureus mixed infection. No history of previous travels.

On admission to the Clinic, he was afebrile. Physical examination revealed presence of cervical and axillar lymphadenopathy and increased bowel movements. Blood tests confirmed an internal inflammation (elevated levels of ESR $36 \mathrm{~mm} / \mathrm{h}$, CRP $31.8 \mathrm{mg} / \mathrm{l}$, WBC $9.17 \mathrm{G} / \mathrm{l}$ ). Because of diarrhea, stool examination was performed. Bacteriological culture according to enteropathogenic bacteria (Salmonella spp., Shigella spp., E.coli ETEC, Yersinia spp., Campylobacter spp., Clostridium difficile) was negative. However, the modified Ziehl-Neelsen staining smears revealed presence of huge amount of Cryptosporidium spp. Oocysts, i.e., 20-50 oocysts/ 10 fields (magnification 1000×), which according to CastroHermida et al. (2001) was classified as a massive infection (Fig. 1). Moreover, elevated fecal calprotectin concentration was detected. Fecal occult blood test (FOBT) was also positive. Electrophoresis showed increased levels of $\alpha 2-, \beta 2-$, and $\gamma$ globulins (15.6/6.9/16.8\%) and shortage of albumins (47\%).

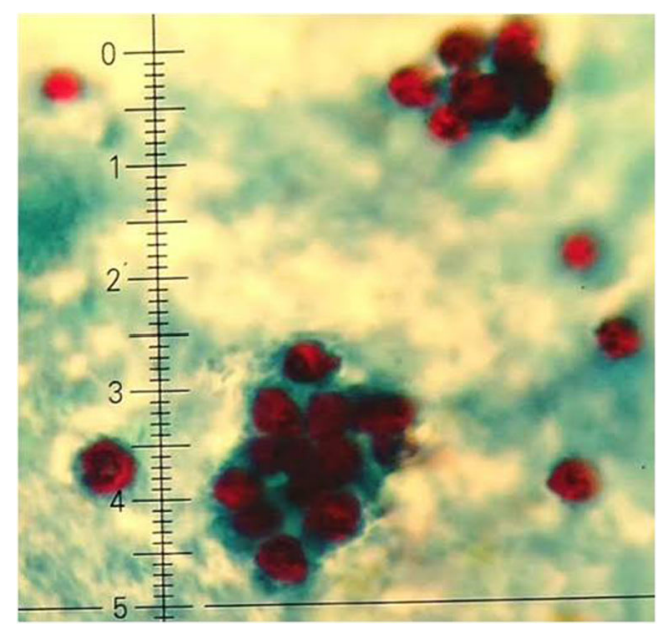

Fig. 1 Microscopical analysis with Ziehl-Neelsen modified staining revealed numerous oocysts of Cryptosporidium spp. in the patient's feces
Because of the massive cryptosporidiosis, the immunodeficiency diagnosis was initiated. Architect CMIA HIV test was negative, HIV-RNA (HIV Cobas TaqScreen MPX version 2.0-Roche) was also negative, normal CD4 count (507/ $\mathrm{mm} 3)$, Treponema RPR test negative, ELISA Toxoplasma gondii (IgM and IgG) negative, ELISA Toxocara IgG negative, HBsAg absent, anti-HCV absent, normal immunoglobulin levels (IgA $1.89 \mathrm{~g} / 1, \operatorname{IgG} 11.43 \mathrm{~g} / 1, \operatorname{IgM} 0.79 \mathrm{~g} / \mathrm{l})$. Colonoscopy (after the patient's permission) detected numerous small afts and shallow ulcers as well as mucosa scarifications.

The histopathological preparations showed incorrect large intestine mucosa architecture with persistent inflammation within submucosa caused by eosinophils and disseminated in lamina propria neutrophils (cryptitis, Fig. 2).

The patient was treated with tilbroquinolonum, trimethoprim/sulphametoxazole $(2 \times 960 \mathrm{mg})$, Saccharomyces boulardii, spiramycin $(3 \times 3.0 \mathrm{mln}$ u. $)$. The patient improved and the parasitic stool test on the last day (after 2 weeks of hospitalization) was negative. Patient was admitted to the Clinic once again after 2 weeks because of relapse of the symptoms (15-20 watery stools, abdominal pain, fever up to $39^{\circ} \mathrm{C}$ ). The stool examination revealed massive Cryptosporidium spp. infection, i.e., 20-30 oocysts/10 fields (magnification 1000×, Castro-Hermida et al. 2001). The presence of other parasites was excluded. Combined therapy with Pyrimethamine $(1 \times 75 \mathrm{mg})$, Azythromycin $(1 \times$ $500 \mathrm{mg})$, and Nitazoxanide $(2 \times 500 \mathrm{mg})$ was initiated. After 10-day treatment, Cryptosporidium eradication was obtained with confirmation after 3 weeks. In control colonoscopy, no signs of inflammatory process were found.

\section{Case 2}

Fifty-six-year-old male hospitalized for 14 days in the Clinic of Tropical and Parasitic Diseases, Poznan, Poland, because of persistent diarrhea, 4-5 watery stools without blood and

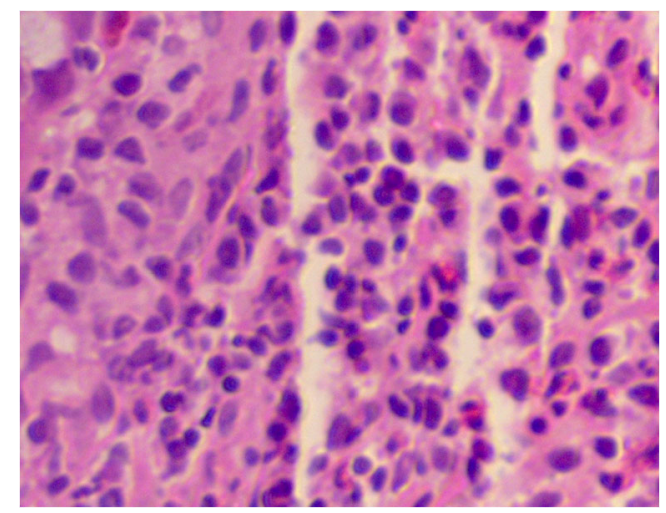

Fig. 2 Large intestine mucosa infiltrated by eosinophils and neutrophils (hematoxylin and eosine staining) 
mucus and fever up to $39^{\circ} \mathrm{C}$. The symptoms appeared 1 year before the admission to the hospital. The patient was treated symptomatically and empirically with amoxicillin, ciprofloxacin, cefuroxime with periodic improvement. He also lost $10 \mathrm{~kg}$ of weight. His medical history of chronic diseases and previous travels was unremarkable.

The physical examination performed on admission revealed presence of numerous enlarged, solid cervical lymphatic nodes, tenderness to palpation in the left subcostal region, and intensive bowel movements. Laboratory findings confirmed acute inflammatory response: increased level of CRP (79.3 ng/l, ESR $47 \mathrm{~mm} / \mathrm{h}$, leukocytosis $16.73 \mathrm{G} / \mathrm{l})$, monocytosis $(8.4 \%)$, neutrocytosis $(81.3 \%)$, and thrombocytosis $(672 \mathrm{G} / \mathrm{l})$. Bacteriological stool tests excluded Salmonella, Shigella, Escherichia coli, and Yersinia spp. infection. Repeated parasitological stool examinations (modified Ziehl-Neelsen staining) revealed presence of numerous Cryptosporidium spp. oocysts, i.e., 20-50 oocysts/10 fields (magnification 1000×; Fig. 3) and elevated level of WBCs and RBCs. High level of fecal calprotectin $(895 \mu \mathrm{g} / \mathrm{l})$ in the stool sample indicated acute bowel inflammation. FOBT was positive. Possible immunodeficiency was excluded, as in the case no. 1.

Colonoscopy (after the patient's permission) performed during hospitalization revealed presence intensive inflammatory mucosal lesions, numerous polyps narrowing the bowel bore, presence of stool-purulent content, and different-shape ulcers (cryptitis). CT scans showed the extraperitoneum lymphadenopathy and intestine wall inflammation (mucosa thickening). Histopathologic sections showed incorrect lamina propria architecture with cryptitis. Chronic, severe inflammation was detected in submucosa. The most abundant type of cells was eosinophils. Disseminated neutrophils, mucosal ulcers covered with granular, and fibrinous changes were also observed.

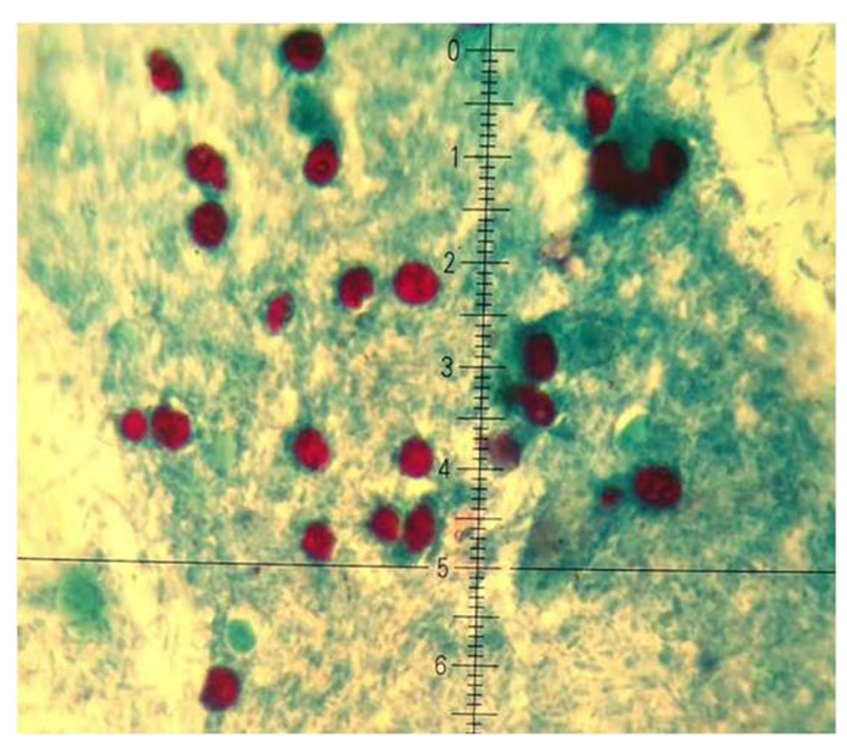

Fig. 3 Cryptosporidium spp. oocyts (modified Ziehl-Neelsen staining)
Combined therapy with trimethoprim/sulphametoxazole and rifaximin was initiated together with symptomatic treatment (20\% albumins, iron supplementation, anti-diarrheal agents). The improvement of the clinical condition was observed, and the patient was discharged home.

After 2 months, the patient was admitted to the Clinic for follow-up. Several parasitic stool examinations were negative. Colonoscopy showed only presence of pseudopolyp and only slight mucosal changes. The histopathological section without abnormalities. Complete normalization of laboratory parameters (CRP-8.7 ng/l, ESR-7 mm/h, WBC-10.1 G/l, PLT$473 \mathrm{G} / \mathrm{l}$, iron $82 \mu \mathrm{g} / \mathrm{dl}$, TIBC $396 \mu \mathrm{g} / \mathrm{dl}$ ) was observed. Parasitological stool examination revealed Cryptosporidium eradication.

\section{Case 3}

Forty-six-year-old woman admitted to the hospital for the differential diagnosis of chronic diarrhea. From 1 month, she had several watery, sometimes bloody stools with high fever up to $39^{\circ}$. Laboratory results showed high CRP level (37.1 ng/l), increased ESR $(33 \mathrm{~mm} / \mathrm{h})$, WBC $(9.1 \mathrm{G} / 1)$. USG of the abdomen cavity did not reveal any abnormalities. Bacteriological stool culture detected Campylobacter jejuni. The patient did not consent to the colonoscopy.

Parasitic stool examination showed massive infection with Cryptosporidium spp. oocysts, i.e., 10-40 oocysts/10 fields (magnification 1000×, Castro-Hermida et al. 2001). High fecal calprotectin level was also detected $(>1000 \mu \mathrm{g} / \mathrm{g})$. The FOBT was positive. The immunodeficiency was excluded like in the previous cases. The patient was treated with trimethoprim/sulphametoxazole $(2 \times 960 \mathrm{mg})$, azythromycin $(1 \times 500 \mathrm{mg})$, rifaximin $(2 \times 400 \mathrm{mg})$. After 10-daytreatment symptoms diminished, stool examinations were negative, and the patient was discharged home.

During follow-up visit in out-patient department, she reported numerous loose stools. Parasitological examinations of the stool samples discovered Cryptosporidium oocysts. Treatment with nitazoxanide was initiated $(3 \times 500 \mathrm{mg}$ for 1 week and $2 \times 500 \mathrm{mg}$ for 3 days). After the treatment, parasite eradication was obtained.

\section{Genotyping of Cryptosporidium}

DNA was extracted from stool samples using a commercial DNA extraction kit (NucleoSpin ${ }^{\circledR}$ Tissue, Macherey-Nagel GmbH \& Co. KG, Düren, Germany) according to the support protocol for genomic DNA stool. To increase the yield of the DNA isolation, five cycles of freezing $\left(-80^{\circ} \mathrm{C}\right)$ and thawing $\left(50{ }^{\circ} \mathrm{C}\right)$ of the stool samples were performed. Because of the 
consistency of the fecal samples, the isolation of each sample was carried out in duplicate, and the DNA samples were concentrated using Eppendorf Concentrator plus (Eppendorf AG, Hamburg, Germany). To identify the Cryptosporidium species, the multiplex allele specific polymerase chain reaction (MAS-PCR) was performed according to Gile et al. with some modifications. (Gile et al. 2002). Briefly, the PCR was performed in a total volume of $20 \mu \mathrm{l}$, consisting of $200 \mathrm{ng}$ isolated DNA, $1.25 \mathrm{U}$ of DreamTaq DNA polymerase (Thermo Fisher Scientific), $2 \mu \mathrm{l}$ of DreamTaq Buffer (10×), $10 \mathrm{pmol}$ of dNTPs, and $20 \mathrm{pmol}$ of forward (CINF) primer and 10 pmol of reverse (CINR, 1R, 2R) primers. The PCR conditions were initial denaturation at $95{ }^{\circ} \mathrm{C}$ for $5 \mathrm{~min}$, followed by 35 cycles of denaturation at $95{ }^{\circ} \mathrm{C}$ for $30 \mathrm{~s}$, annealing at $50{ }^{\circ} \mathrm{C}$ for $30 \mathrm{~s}$, and elongation at $72{ }^{\circ} \mathrm{C}$ for $1 \mathrm{~min}$, with final elongation at $72{ }^{\circ} \mathrm{C}$ for $10 \mathrm{~min}$. Amplified products were subjected to electrophoreses in a $2 \%$ agarose gel. The results were confirmed in three independent reaction sets.

The MAS-PCR, used for the identification of Cryptosporidium species, amplifies the dihydrofolate reductase (DHFR) gene with one sense and three antisense primers (CINF and CINR, 1R, 2R, respectively). Primer-pairs CINF and CINR amplify a 575 bp region of the DHFR gene, specific for Cryptosporidium spp., and simultaneously confirm the infection with this pathogen. Additionally, the primer set CINF and $1 \mathrm{R}$ amplifies a $357 \mathrm{bp}$ region, specific for C. hominis, while the primer-pair CINF and $2 \mathrm{R}$ gives the product with $190 \mathrm{bp}$ size, specific for C. parvum. In all three cases, the $575 \mathrm{bp}$ band was detected, confirming the infection with Cryptosporidium spp. Additionally, the band with $357 \mathrm{bp}$ size was observed in all three cases, suggesting the infection with Cryptosporidium hominis (Fig. 4). The specific band for C. parvum was not detected in any sample. As a negative control, we used the stool sample from a patient without cryptosporidiosis, and any band was not observed. The stool from the patient with previously diagnosed cryptosporidiosis was taken as a positive control (Fig. 4).

\section{Discussion}

Chronic diarrhea lasts longer than three and is rarely caused by the infectious agents (Sandhu and Surawicz 2012). Protozoa of the genus Cryptosporidium are considered as one of the etiologic agents of persistent diarrheal illness in immunocompromised patients (Clemente et al. 2000; Werneck-Silva and Bedin Prado 2009). However, they are more often detected also in HIV-negative humans, as a cause of travelers' diarrhea, in immunocompetent children or in man infected with the hedgehog genotype (Kłudkowska et al. 2017; Tallant et al. 2016; Kváč et al. 2014).

The main clinical symptoms observed in the infected patients are watery diarrhea, abdominal pains, and mild fever. Interestingly, non-intestinal sequelae of cryptosporidiosis can be also present, i.e., arthropathies, stiffness in the lumbosacralis region, eye pains, and headaches (Hunter et al. 2014). In one of the described patients painful, inflamed joints were also observed. According to the literature, the extraintestinal signs occur even in 30\% of infected individuals (LopezVelez et al. 1995). Moreover, hematogenous Cryptosporidium spread with the blood vessels was observed (Gentile et al. 1987) and negative changes in the liver and the heart were detected. The intestinal parasitic infections may bring about functional impairments of internal organs leading to long-term consequences (Beier et al. 2006). Cryptosporidium oocysts were found in the trachea, sputum, and sinus aspirates. Cryptosporidiosis in the upper respiratory tract might cause inflammation in the sinuses, larynx, and nasal mucosa (Abdali et al. 2018). However, all these complications were detected in HIV-infected individuals. The clinical course is mostly selflimiting. However, in cases of massive or mixed infections, persistent symptoms are observed, and patients require combined anti-parasitic and also anti-bacterial treatment.

Massive cryptosporidiosis described in our three patients was not related to previous travels into tropical regions. They also deny having contact with any animals and had normal diet. Moreover, the stools of other roommates were examined and were parasitologically negative. Immunodeficiency diagnostics
Fig. 4 Genotyping of Cryptosporidium spp.

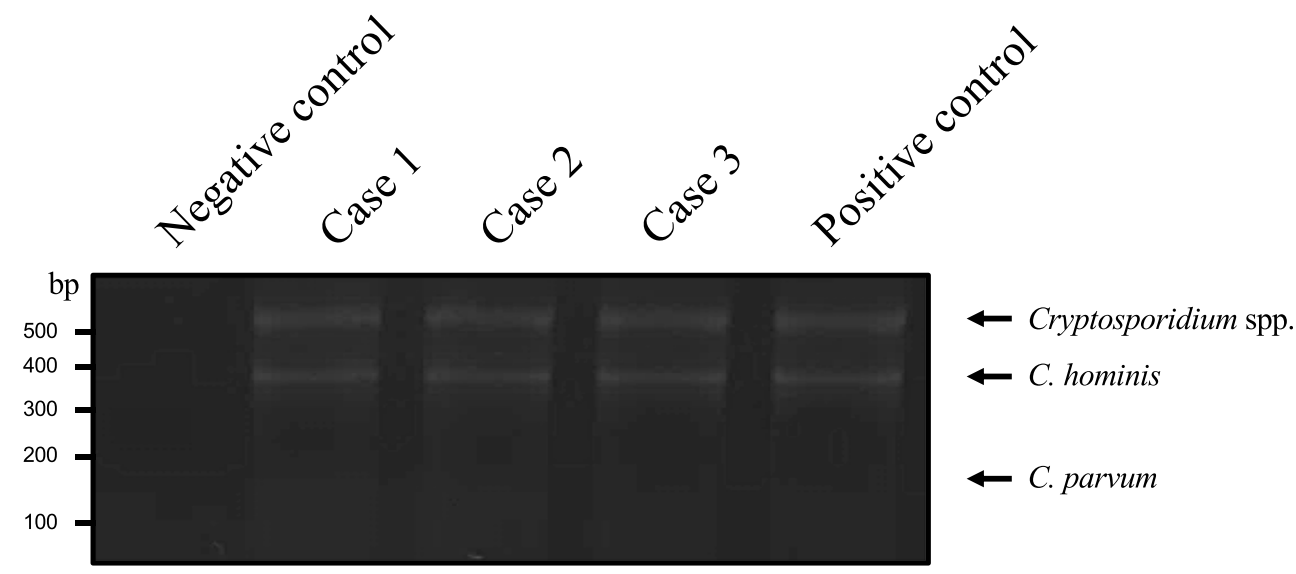


did not reveal any abnormalities. The lesions discovered during colonoscopy were related to massive cryptosporidiosis, since long-lasting and severe infection leads to lamina propria damage (Abdou et al. 2013). In the literature, you can find descriptions of endoscopic and histopathological changes in the stomach mucosa caused by Cryptosporidium spp., but they are mainly referred to immunocompromised persons (Clemente et al. 2000; Mohammadpour et al. 2016). Histopathologic features associated to Cryptosporidium infection include stomach infiltrates, villus lining in the duodenum, cryptitis caused by massive eosinophils concentration, and epithelial apoptosis in the colon (WerneckSilva and Bedin Prado 2009). However, most of these findings concern to the HIV-positive patients.

The cases described in our study indicate that coccidian parasites might be responsible for gastrointestinal pathology resulting with chronic diarrhea also in HIV-negative persons. Massive infections should be treated because they lead to huge intestine damage, which could be responsible for prolonged even life-threatening symptoms especially in children, teenagers, and malnourished individuals. Moreover, they can influence on gut microbiota. The current therapeutic options for cryptosporidiosis are limited and only partially effective. In two out of three described cases, the treatment with trimethoprim/sulphametoxazole was insufficient. Nitazoxanide and azithromycin have better effectiveness for cryptosporidiosis treatment in humans (Lee et al. 2017).

To sum up, Cryptosporidium infection must be taken under consideration in differential diagnosis of persistent diarrhea in individuals without HIV infection. Additionally, it should be highlighted that persistent Cryptosporidium hominis infection can be responsible for enhanced lamina propria and submucosa alterations within large intestine, which strictly connects to prolonged intestinal symptoms in patients without HIV infection. Moreover, Cryptosporidium infection may also lead to extraintestinal abnormalities, i.e., extraperitoneal lymphadenopathy as well as generalized arthritis, and thus, patients suffering from such symptoms should be carefully examined to detect coccidian parasites. All these statements indicate that parasite's eradication leads to symptom disappearance as well as improvement of histopathological mucosa alterations.

Open Access This article is distributed under the terms of the Creative Commons Attribution 4.0 International License (http:// creativecommons.org/licenses/by/4.0/), which permits unrestricted use, distribution, and reproduction in any medium, provided you give appropriate credit to the original author(s) and the source, provide a link to the Creative Commons license, and indicate if changes were made.

\section{References}

Abdali A, Ghaffarifar F, Pirestani M (2018) Neglected risk factors of childhood morbidity and mortality caused by cryptosporidium infection. Lancet Glob Health 6(10):e1068. https://doi.org/10.1016/ s2214-109X(18)30377-2
Abdou AG, Harba NM, Afifi AF, Elnaidany NF (2013) Assessment of Cryptosporidium parvum infection in immunocompetent and immunocompromised mice and its role in triggering intestinal dysplasia. Int J Infect Dis 17(8):e593-e600

Beier TV, Sidorenko NV, Anatskaia OV (2006) Intestinal cryptosporidiosis at an early age and its negative consequences. Med Parazitol (Mosk) (1):3-7

Bouzid M, Hunter PR, Chalmers RM, Tyler KM (2013) Cryptosporidium pathogenicity and virulence. Clin Microbiol Rev 26(1):115-134. https://doi.org/10.1128/CMR.00076-12

Castro-Hermida JA, Quilez-Cinca J, Lopez-Bernad F, Sanchez-Acedo C, Freire-Santos F, Ares-Mazas E (2001) Treatment with betacyclodextrin of natural Cryptosporidium parvum infections in lambs under fiels conditions. Int J Parasitol 31(10):1134-1137

Clemente CM, Caramon CA, Padula P, Rodriques MA (2000) Gastric cryptosporidiosis as a clue for the diagnosis of the acquired immunodeficiency syndrome. Arg Gastroenterol 37(3):180-182

Domenech E, Amoros I, Moreno Y, Alonso IL (2018) Cryptosporidium and giardia safety margin increase in leafy green vegetables irrigated with treated waste water. Int J Hyg Environ Health 221(1):112-119

DuPont HL (2016) Persistent diarrhea: a clinical review. Jama. 315(24): $2712-2723$

Fill MA, Lloyd J, Chakraverty T, Sweat D, Manners J, Garman K, Hlavsa MC, Roellig DM, Dunn JR, Schaffner W, Jones TF (2017) Cryptosporidiosis outbreak associated with a single hotel. J Environ Health 79(9): 16-22

Gentile $\mathrm{G}$ et al (1987) Colonic invasion as possible route of extraitestinal cryptosporidiosis. Am J Med 82(3):574-575

Gile M, Warhurst DC, Webster KA, West DM, Marshall JA (2002) A multiplex allele specific polymerase chain reaction (MAS-PCR) on the dihydrofolate reductase gene for the detection of Cryptosporidium parvum genotypes 1 and 2. Parasitology 125(Pt 1):35-44

Hall V, Taye A, Walsh B, Maquire H, Dave J, Wright A, Anderson C, Crook P (2017) A large outbreak of gastrointestinal illness at open water swimming event in the river Thames London. Epidemiol Infect 145(6):1246-1255

Hlavsa MC, Roellig DM, Seabolt MH, Kahler AM, Murphy JL, McKitt TK, Geeter EF, Dawsey R, Davidson SL, Kim TN, Tucker TH, Iverson SA, Garrett B, Fowle N, Collins J, Epperson G, Zusy S, Weiss JR, Komatsu K, Rodriguez E, Patterson JG, Sunenshine R, Taylor B, Cibulskas K, Denny L, Omura K, Tsorin B, Fullerton KE, Xiao L (2017) (2016) using molecular characterization to support investigations of aquatic facility-associated outbreaks of cryptosporidiosis - Alabama, Arizona, and Ohio. MMWR Morb Mortal Wkly Rep 66:493-497

Hunter PR, Hughes S, Woodhouse S, Raj N, Syed Q, Chalmers RM, Verlander NQ, Goodacre J (2014) Health sequelae of human cryptosporidiosis in immunocompetent patients. Clin Infect Dis 39(4): $504-510$

Kaniyarakkal V, Mundangalam N, Maarkath AP, Mathew S (2016) Intestinal parasite profile in the stool of HIV positive patients in relation to immune status and comparison of various diagnostic techniques with special reference to cryptosporidium at a tertiary Care Hospital in South India. Adv Med 2016:3564359

Kłudkowska M, Pielok Ł, Frąckowiak K, Paul M (2017) Intestinal coccidian parasites as an underestimated cause of travellers' diarrhoea in polish immunocompetent patients. Acta Parasitol 62(3): $630-638$

Kváč M, Saková K, Květoňová D, Kicia M, Wesołowska M, McEvoy J, Sak B (2014) Gastroenteritis caused by the Cryptosporidium hedgehog genotype in an immunocompetent man. J Clin Microbiol 52(1): 347-349

Lantermier F, Amazzough K, Favennec L, Mamas-Buneel M (2017) Cryptosporidium spp. infection in solid organ transplantation: the Nationwide "Transcrypto" study. Transplantation 101(4):826-830 
Lee S, Harwood M, Girouard D, Meyers MJ, Campbell MA, Beomer G, Tzpori S (2017) The therapeutic efficacy of azithromycin and nitazoxanide in the acute pig model of Cryptosporidium hominis. PLoSone 12(10):e0185906

Lopez-Velez R et al (1995) Intestinal and extraintestinal cryptosporidiosis in AIDS patients. Eur J Clin Microbiol Infect Dis 14(8):677-681

Mohammadpour I, Bozag-Chalati F, Motazedian MA (2016) Molecular characterization and phylogenetic analysis of microsporidia and Cryptosporidium spp. in patients with multiple bowel biopsies from Fars Province. Iran Ann Parasitol 62(4):321-330

Osman M, El Safadi D, Benamrouz-Vanneste S, Cian A, Moriniere R, Gantois N, Delgado-Viscogliosi P, Guyot K, Bosc S, Chabé M, Petit T, Viscogliosi E, Certad G (2017) Prevalence, transmission, and host specificity of Cryptosporidium spp. in various animal group from two French zoos. Parasitol Res 116(12):3419-3422

Sandhu DK, Surawicz C (2012) Update on chronic diarrhea: a runthrough for the clinician. Curr Gastroenterol Rep 14:421-427

Shrivastava AK, Kumar S, Mohakud NK, Suar M, Sahu PS (2017a) Multiple etiologies of infectious diarrhea and concurrent infections in a pediatric outpatient-based screening study in Odisha, India. Gut Pathog 11(9): 16

Shrivastava AK, Kumar S, Snith WA, Sahn PS (2017b) Revisiting the global problem of cryptosporidiosis and recommendations. Trop Parasitol 7(1):8-17
Squire SA, Ryan U (2017) Cryptosporidium and giardia in Africa : current and future challenges. Parasit Vectors 10(1):195

Tallant C, Huddleston P, Alshenberi A, Misra S (2016) Acute. Severe cryptosporidiosis in an immunocmpetent pediatric patient. Clin Pract 6(2):837

Toledo RP, Martins FD, Ferrera FP, de Almeida JC, Garcia JL (2017) Cryptosporidium spp. and Giardia spp. in feces and water and the associated exposure factors on dairy farms. PloSOne 12(4): e0175311

Werneck-Silva AL, Bedin Prado I (2009) Role of upper endoscopy in diagnosing opportunistic infections in human immunodeficiency virus-infected patients. World J Gastroenterol 15(9):1050-1056

Yu Z, Ruan Y, Ahon M, Chen S, Zhang Y, Wang L, Zhu G, Yu Y (2018) Prevalence of intestinal parasites in companion dogs with diarrhea in Beijing, China and genetic characteristics of Giardia and Cryptosporidium species. Parasitol Res 117(1):35-43

Zehedi A, Paparini A, Jian F, Robertson I, Ryan U (2015) Public health significance of zoonotic Cryptosporidium species in wildlife: critical insight into better drinking water management. Int J Parasitol Wildlife 5(1):88-109

Publisher's note Springer Nature remains neutral with regard to jurisdictional claims in published maps and institutional affiliations. 